PROCEEDINGS OF THE

AMERICAN MATHEMATICAL SOCIETY

Volume 128, Number 6, Pages 1845-1851

S 0002-9939(99)05172-2

Article electronically published on October 18, 1999

\title{
CHERN NUMBERS OF CERTAIN LEFSCHETZ FIBRATIONS
}

\author{
ANDRÁS I. STIPSICZ
}

(Communicated by Ronald A. Fintushel)

\begin{abstract}
We address the geography problem of relatively minimal Lefschetz fibrations over surfaces of nonzero genus and prove that if the fiber-genus of the fibration is positive, then $0 \leq c_{1}^{2} \leq 5 c_{2}$ (equivalently, $0 \leq c_{1}^{2} \leq 10 \chi_{h}$ ) holds for those symplectic 4-manifolds. A useful characterization of minimality of such symplectic 4-manifolds is also proved.
\end{abstract}

\section{INTRODUCTION}

It is a longstanding problem of 4-dimensional manifold theory to determine which simply connected topological 4-manifolds support irreducible smooth structures. (A smooth 4-manifold $X$ is called irreducible if for every decomposition $X=X_{1} \# X_{2}$ either $X_{1}$ or $X_{2}$ is homeomorphic to the 4 -sphere $S^{4}$.) Using Seiberg-Witten theory it was shown that simply connected minimal complex surfaces are irreducible - the determination of the homeomorphism types of those (frequently called the geography problem of complex surfaces) has been essentially answered. Coupling theorems of Donaldson [D1] and Freedman [F] we get that the homeomorphism type of a smooth simply connected 4-manifold $X$ is determined by the fact whether it is spin, by its signature $\sigma(X)$ and by its Euler characteristic $e(X)$. (Instead of the latter two numerical invariants we will rather use $\chi_{h}(X)=\frac{1}{4}(\sigma(X)+e(X))$ and $c_{1}^{2}=3 \sigma(X)+2 e(X)$.) It has been proved that if $S$ is a minimal, simply connected complex surface, then either $c_{1}^{2}(S)=0$ (and $\chi_{h}(S) \geq 0$ is arbitrary) or $c_{1}^{2}(S)>0$ and $2 \chi_{h}(S)-6 \leq c_{1}^{2}(S) \leq 9 \chi_{h}(S)$ [BPV]. The latter two inequalities are called the Noether- and Bogomolov-Miyaoka-Yau $(B M Y)$ inequalities. Note that $c_{1}^{2}(S) \leq$ $9 \chi_{h}(S)$ is equivalent to $c_{1}^{2}(S) \leq 3 c_{2}(S)$ and obviously $c_{2}(S)=e(S)$ holds. (Persson and his collaborators [P], [PPX] essentially showed that the above mentioned are the only constraints.)

Remark 1.1. Although in the above discussion we restricted our attention to the simply connected case, the inequality $c_{1}^{2}(S) \leq 3 c_{2}(S)$ is, in fact, satisfied by complex surfaces (with $b_{2}^{+}(S)>1$ ) of arbitrary fundamental groups.

Because of their deep and surprising connections with Seiberg-Witten theory, the study of symplectic 4-manifolds became a central subject of 4-manifold theory. Consequently, the above geography question can be extended to the symplectic

Received by the editors June 29, 1998 and, in revised form, July 14, 1998.

1991 Mathematics Subject Classification. Primary 57R99, 57M12.

Key words and phrases. 4-manifolds, Lefschetz fibrations, geography problem.

Supported by the Magyary Zoltán Foundation and OTKA.

(C)2000 American Mathematical Society 
case; note also that minimality can be easily adapted in the symplectic category. By fundametal results of Taubes T2 we have that minimal (simply connected) symplectic 4-manifolds with $b_{2}^{+}>1$ are irreducible and satisfy $c_{1}^{2} \geq 0[\mathrm{Ko}]$. (For more about results concerning the geography of symplectic 4-manifolds see [S1] and GS.) The symplectic version of the BMY-inequality is, however, still missing.

Remark 1.2. Note that since by definition $c_{1}^{2}(X)+c_{2}(X)=12 \chi_{h}(X)$, for manifolds with $c_{2}(X)=e(X)=2-2 b_{1}(X)+b_{2}(X) \geq 0$ we have $c_{1}^{2}(X) \leq 12 \chi_{h}(X)$. If, in addition, we assume that the 4-manifold $X$ is simply connected, then $c_{1}^{2}(X) \leq$ $5 c_{2}(X)$ (equivalently, $c_{1}^{2}(X) \leq 10 \chi_{h}(X)$ ) can be easily shown (see Remark 2.4). Note that for all known irreducible 4 -manifolds $c_{1}^{2} \geq 0$ holds; by reversing the orientation this gives that for all known irreducible 4-manifolds we have $c_{1}^{2} \leq 4 c_{2}$ (equivalently, $c_{1}^{2} \leq \frac{48}{5} \chi_{h}$ ). The knowledge of a sharp constant $b$ in an inequality of the type $c_{1}^{2} \leq b \cdot c_{2}$ (equivalently, $c_{1}^{2} \leq \frac{12 b}{b+1} \chi_{h}$ ) in the irreducible and in the symplectic case would be of fundamental importance. We only note here that the conjecture that $c_{1}^{2} \geq 0$ holds for every simply connected irreducible 4-manifold is called the $\frac{3}{2}$-conjecture; the proof of it would already imply the solution of the $\frac{11}{8}$-conjecture.

In the present note we address a similar question for Lefschetz fibrations; we will show the following:

Theorem 1.3. If a 4-manifold $X$ admits a Lefschetz fibration $f: X \rightarrow \Sigma$ with generic fiber $F$, and $g(F), g(\Sigma)>0$, then $c_{1}^{2}(X) \leq 5 c_{2}(X)$. (Here $g(F)$ and $g(\Sigma)$ denote the genus of the fiber and the base of the Lefschetz fibration.)

Note that such 4-manifolds necessarily have $b_{1}(X) \geq 2$; hence the reasoning given in Remark 2.4 does not apply. Since in geography we are most concerned about minimal manifolds, we will prove the following simple characterization.

Theorem 1.4. A Lefschetz fibration $f: X \rightarrow \Sigma$ with $g(\Sigma)>0$ is minimal iff it is relatively minimal. (For the definition of relatively minimal Lefschetz fibrations see Definition [2.1)

In conclusion, as a consequence of the above theorems we derive

Theorem 1.5. If $f: X \rightarrow \Sigma$ is a relatively minimal Lefschetz fibration and $g(F)$, $g(\Sigma)>0$, then

$$
0 \leq c_{1}^{2}(X) \leq 5 c_{2}(X) .
$$

Remarks 1.6. (1) By a theorem of Gompf [G], [GS] most Lefschetz fibrations admit symplectic structures - the exceptions all have fiber-genus 1 and are blow-ups of torus fibrations with no critical points; these exceptions can be analyzed by other methods. Hence Theorem 1.3 deals with a part of the geography problem for symplectic 4-manifolds having nontrivial fundamental groups.

(2) By a recent result of Donaldson [D2] each symplectic 4-manifold $X$ can be blown up to admit a Lefschetz fibration. These fibrations are over $\mathbb{C P}^{1}$; hence our theorem does not cover this general case.

(3) Using various standard constructions [FS2, S2 it can be shown that most pairs $(a, b) \in \mathbb{N} \times \mathbb{N}$ satisfying $b \leq 3 a$ correspond to relatively minimal Lefschetz fibration via $a=c_{2}$ and $b=c_{1}^{2}$. No examples of a Lefschetz fibration (or symplectic 4-manifold) $X$ with $c_{1}^{2}(X)>3 c_{2}(X)$ have been found yet. 


\section{LEFSCHETZ FIBRATIONS}

We begin with a brief summary of the basics of Lefschetz fibrations.

Definition 2.1. Suppose that $X$ and $\Sigma$ are smooth, closed, oriented manifolds of dimensions 4 and 2 respectively. A smooth map $f: X \rightarrow \Sigma$ is called a Lefschetz fibration if each critical point of $f$ admits an orientation-preserving coordinate chart $\varphi_{\alpha}: U_{\alpha} \rightarrow V_{\alpha} \subset \mathbb{C}^{2}$ on which $f$ is of the form $f\left(z_{1}, z_{2}\right)=z_{1}^{2}+z_{2}^{2}$. The (finite) set of critical points is denoted by $C$. Fibers disjoint from $C$ are called generic fibers, while the ones intersecting $C$ nontrivially are the singular fibers. The genus $g(F)$ of the generic fiber $F$ is called the genus of the Lefschetz fibration. A Lefschetz fibration is relatively minimal if there is no fiber which contains a sphere of self-intersection -1 .

Examples 2.2. (1) Genus-0 Lefschetz fibrations are easy to classify: if such a fibration is relatively minimal, then it does not contain critical points at all. Consequently, a relatively minimal genus-0 Lefschetz fibration is simply an $S^{2}$-fibration over $\Sigma$. On the other hand, $S^{2}$-fibrations are easy to describe: depending on the parity of the intersection form $Q_{X}$, the 4-manifold $X$ is diffeomorphic either to $S^{2} \times \Sigma$ or to $S^{2} \tilde{\times} \Sigma$. Easy computation shows that in both cases $c_{1}^{2}(X)=8(1-g(\Sigma))$ and $c_{2}(X)=4(1-g(\Sigma))$; in particular, $c_{1}^{2}(X)=2 c_{2}(X)$ (equivalently, $c_{1}^{2}(X)=8 \chi_{h}(X)$ ) holds. Note that the above case is excluded from Theorem 1.3. In the following we will always assume that the genus of the Lefschetz fibration under study is positive.

(2) The genus-1 case has been extensively studied by Matsumoto [M]; in particular, it has been shown that if $f: X \rightarrow \Sigma$ is a genus-1 Lefschetz fibration, then $c_{1}^{2}(X)=0$ and $c_{2}(X) \geq 0$; see also [GS.

(3) Note also that if the relatively minimal Lefschetz fibration $f: X \rightarrow \Sigma$ has no critical points (i.e., $X$ is a surface bundle over $\Sigma$ ), then $g(F), g(\Sigma)>0$ implies $\pi_{2}(X)=0$; consequently, in particular, such $X$ necessarily does not contain a sphere with square -1 .

Lemma 2.3. For a 4-manifold $X$ we have $c_{1}^{2}(X)=5 c_{2}(X)+6\left(b_{1}(X)-b_{2}^{-}(X)-1\right)$.

Proof. By definition we have $c_{1}^{2}(X)-2 c_{2}(X)=3 \sigma(X)=3\left(b_{2}^{+}(X)-b_{2}^{-}(X)\right)=$ $3\left(c_{2}(X)+2 b_{1}(X)-2 b_{2}^{-}(X)-2\right)=3 c_{2}(X)+6\left(b_{1}(X)-b_{2}^{-}(X)-1\right)$, proving the lemma.

Remark 2.4. Note that if $X$ is simply connected (in fact, $b_{1}(X) \leq 1$ suffices), then Lemma 2.3 implies $c_{1}^{2}(X) \leq 5 c_{2}(X)$.

Proposition 2.5. If $f: X \rightarrow \Sigma$ is a genus- $k$ Lefschetz fibration, $k \geq 2$ and $l=$ $g(\Sigma) \geq 1$, then $b_{1}(X) \leq 2 k+2 l$ and $b_{2}^{-}(X) \geq 2 k l+1$.

Proof. The estimate on $b_{1}(X)$ follows trivially from the fact that $X$ admits a handle decomposition with $2 k+2 l$ 1-handles. This can be seen in the following way. Assume that $f(C) \subset D^{2} \subset \Sigma$ where $D^{2}$ is an embedded disk (i.e., over $\Sigma-D^{2}$ our Lefschetz fibration is a fiber bundle); then $f^{-1}\left(D^{2}\right)$ can be built from $F \times D^{2}$ by gluing 2 -handles only [Ka], GS]. Note that $F \times D^{2}$ admits an obvious handle decomposition involving $2 k$ 1-handles (and a single 2-handle). Now to complete the handle decomposition of $X$ we have to add one 1-handle, $2 k 2$-handles and a 3 -handle to $f^{-1}\left(D^{2}\right)$ for each 1-handle of $\Sigma[\mathrm{GS}$. (The decomposition then will be finished by gluing another copy of $F \times D^{2}$ on the top of the previous handles, meaning the addition of one 2-handle, $2 k$-handles and a 4 -handle.) Since $\Sigma$ is 
of genus $l$, the above procedure requires the addition of $2 l$ 1-handles to $f^{-1}\left(D^{2}\right)$; consequently it proves $b_{1}(X) \leq 2 k+2 l$.

For proving the estimate on $b_{2}^{-}(X)$, we will find $2 k l$ pairs of linearly independent tori in $X$ each of square 0 such that each pair forms a hyperbolic pair $H=\left[\begin{array}{ll}0 & 1 \\ 1 & 0\end{array}\right]$ in the intersection form of $X$. To see this, fix $a_{1}, b_{1}, \ldots, a_{l}, b_{l}$ and $\alpha_{1}, \beta_{1}, \ldots, \alpha_{k}, \beta_{k}$ circles in $\Sigma$ and $F$ respectively, satisfying $a_{i} \cap a_{j}=\emptyset, a_{i} \cap b_{j}=\emptyset$ (for $i \neq j$ ) and $a_{i} \cap b_{i}=\{p t$.$\} (and similar relations for \alpha$ 's and $\beta$ 's). (The obvious circles on a surface of genus $l$ and $k$ will provide such collections.) Next, push each circle radially away from itself to get $a_{1}^{\prime}, b_{1}^{\prime}, \ldots, a_{l}^{\prime}, b_{l}^{\prime}$ and $\alpha_{1}^{\prime}, \beta_{1}^{\prime}, \ldots, \alpha_{k}^{\prime}, \beta_{k}^{\prime}$. Obviously $a_{i}^{\prime} \cap a_{j}^{\prime}=a_{i}^{\prime} \cap a_{j}=\emptyset, a_{i}^{\prime} \cap b_{j}^{\prime}=a_{i}^{\prime} \cap b_{j}=\emptyset(i \neq j)$ and $a_{i}^{\prime} \cap b_{i}=\{p t\},. a_{i}^{\prime} \cap b_{i}^{\prime}=\{p t$. (and similar relations for $\alpha^{\prime}$ and $\beta^{\prime}$ ). Now the tori $\left\{a_{i} \times \alpha_{j}, b_{i} \times \beta_{j}, a_{i}^{\prime} \times \beta_{j}^{\prime}, b_{i}^{\prime} \times \alpha_{j}^{\prime} \mid i=\right.$ $1, \ldots, l ; j=1, \ldots, k\}$ give the appropriate collection of homology elements in $X$. Extending this collection to a basis of $H_{2}(X ; \mathbb{Z})$ we get a presentation of the intersection form $Q_{X}$ as $2 k l \cdot H \oplus Q^{\prime}$. It is easy to see that the above tori can be chosen to be disjoint from a generic fiber of the Lefschetz fibration. Since the fiber is homologically essential - because $g(F) \geq 2$, hence it is a symplectic submanifold of the symplectic 4-manifold $X$ (cf. [G] and Remark [1.6(1)) - and its self-intersection is 0 , we conclude that $Q^{\prime}$ is not positive definite. Consequently $b_{2}^{-}(X)=b_{2}^{-}\left(Q_{X}\right)=b_{2}^{-}(2 k l \cdot H)+b_{2}^{-}\left(Q^{\prime}\right)=2 k l+b_{2}^{-}\left(Q^{\prime}\right) \geq 2 k l+1$, which inequality proves the proposition.

Corollary 2.6. If $f: X \rightarrow \Sigma$ is a Lefschetz fibration as in Proposition [2.5, then $b_{1}(X) \leq b_{2}^{-}(X)+1$; hence $c_{1}^{2}(X) \leq 5 c_{2}(X)$.

Proof. Since (by our assumptions on $k$ and $l$ ) we have $0 \leq(k-1)(l-1)$, the inequality $2 k+2 l \leq 2 k l+2$ follows. By Proposition 2.5 this implies $b_{1}(X) \leq$ $b_{2}^{-}(X)+1$, which (by Lemma 2.3) gives $c_{1}^{2}(X)=5 c_{2}(X)+6\left(b_{1}(X)-b_{2}^{-}(X)-1\right) \leq$ $5 c_{2}(X)$.

\section{Minimality of Lefschetz fibrations}

This final section is devoted to the proofs of Theorems 1.4 and 1.5. Obviously, if $X$ is minimal, then any Lefschetz fibration on $X$ is relatively minimal (since there is no sphere in $X$ with self-intersection -1 at all). Note that if $f: X \rightarrow \Sigma$ is a torus bundle, then Example 2.2(3) proves Theorem 1.4 in that case. Hence in the following we will assume that $f: X \rightarrow \Sigma$ is not a torus bundle, cf. also Remark 1.6(1). Similarly, we will assume that the genus of the generic fiber is positive; hence $b_{2}^{+}(X)>1$ (cf. Example 2.2(1)). Before proving the converse of Theorem 1.4 in general (i.e., that a relatively minimal Lefschetz fibration with $g(\Sigma)>0$ has minimal total space), however, we need a little preparation.

A Lefschetz fibration $f: X \rightarrow \Sigma$ can be perturbed to another Lefschetz fibration $f^{\prime}: X \rightarrow \Sigma$ with critical points $C^{\prime}$ such that $f^{\prime}$ is injective on $C^{\prime}$ (i.e., a singular fiber contains a unique critical point). It is easy to see that this perturbation can be done locally around each singular fiber; consequently $f^{\prime}$ is relatively minimal iff $f$ is. Hence, without loss of generality, we might assume that $f: X \rightarrow \Sigma$ is injective on the set $C$ of critical points.

A fiber $f^{-1}(t)(t \in \Sigma)$ is an immersion of a (possibly disconnected) Riemann surface $R$. In case $f^{-1}(t)$ is regular, or singular and the corresponding vanishing cycle is nonseparating, $R$ turns out to be connected. If the vanishing cycle corresponding to a singular fiber is separating, then the fiber is the image of the union 
of two connected Riemann surfaces $R_{1} \cup R_{2}$; in this case we have $\left[R_{1}\right] \cdot\left[R_{2}\right]=1$ and $\left[R_{i}\right]^{2}=-1(i=1,2)$ in $X$. (Recall that we assumed the injectivity of $f$ on $C$. For proofs of the above statements see [Ka] and [GS].) Consequently:

Lemma 3.1. Suppose that $f: X \rightarrow \Sigma$ is a Lefschetz fibration and $f$ is injective on $C$. If $R$ is a Riemann surface embedded in a fiber of $f$, then either $[R]^{2}=0$ or $[R]^{2}=-1$ in $X$.

For a Lefschetz fibrations $f: X \rightarrow \Sigma$ we can equip $X$ with a symplectic structure $\omega$ (see G], GS]) - note that the blown up torus bundle case has been treated separately, so can be excluded here (cf. Remark 1.6). In fact, following the proof of Gompf, we can find an $\omega$-compatible almost-complex structure $J$ on $X$ and an almost-complex structure $J_{0}$ on $\Sigma$ such that the map $f: X \rightarrow \Sigma$ is pseudoholomorphic, i.e., $J_{0} \circ d f=d f \circ J$.

Remark 3.2. Gompf proves the existence of a symplectic structure by first fixing symplectic forms on the complex coordinate charts around points of $C$ and then extending those forms to a global, closed (not necessarily nondegenerate) 2-form $\eta$ on $X$ which restricts to a symplectic form on each fiber. Now taking appropriate (compatible) almost-complex structures along the fibers, and pulling back an arbitrary almost-complex structure $J_{0}$ of $\Sigma$ to the $\eta$-orthogonals of the tangents of the fibers in each tangent space $T_{p} X$ via $f$, we get an appropriate almost-complex structure $J$ which is compatible with the symplectic structure provided by the construction of Gompf. For further details see [GS].

Applying the connection between Seiberg-Witten theory and symplectic topology, we will prove a theorem concerning symplectic 4-manifolds; this result will conclude the proof of Theorem [1.4.

Theorem 3.3. Suppose that $(X, \omega)$ is a symplectic 4-manifold with a compatible almost-complex structure $J$ and the homology class $e \in H_{2}(X ; \mathbb{Z})$ with $e^{2}=-1$ can be represented by a sphere. Under the above hypotheses there is an embedded pseudo-holomorphic sphere in $(X, J)$.

For the proof we have to recall a few facts about the Seiberg-Witten invariants of $X$ (cf. $[\mathrm{W}]$ ) and the relation of those with pseudo-holomorphic curves in $X$.

Theorem 3.4 (Taubes, [T1], [T2]). If $(X, \omega)$ is a symplectic 4-manifold and $b_{2}^{+}(X)$ $>1$, then $S W_{X}\left( \pm c_{1}(X, \omega)\right)= \pm 1$. If for $c \in H_{2}(X ; \mathbb{Z})$ the Seiberg-Witten invariant $S W_{X}\left(c_{1}(X, \omega)+2 P D(c)\right) \neq 0$, then the Gromow invariant $G r_{X}(c)$ is nonzero as well.

Applying the generalized adjunction formula [KM], [MSzT], the nontriviality of the Seiberg-Witten invariant of a symplectic 4-manifold implies the following:

Corollary 3.5. If $X$ is a symplectic 4-manifold with $b_{2}^{+}(X)>1$ and $S \subset X$ is a homologically essential embedded sphere, then $[S]^{2}<0$.

Theorem 3.6 (The blow-up formula, [FS1]). If $M$ is a smooth 4-manifold, then $S W_{M}(c)= \pm S W_{M \# \overline{\mathbb{C P}^{2}}}(c \pm P D(e))$, where $e$ denotes the generator of the $\overline{\mathbb{C P}^{2}}$ summand in $H_{2}\left(M \# \overline{\mathbb{C P}^{2}} ; \mathbb{Z}\right)$ and $c \in H^{2}(M ; \mathbb{Z}) \subset H^{2}\left(M \# \overline{\mathbb{C P}^{2}} ; \mathbb{Z}\right)$.

Note that if a symplectic 4-manifold $X$ contains an embedded sphere of selfintersection -1 , then $X=X^{\prime} \# \overline{\mathbb{C P}^{2}}$ where $X^{\prime}$ is symplectic and $c_{1}(X, \omega)=$ $c_{1}\left(X^{\prime}, \omega^{\prime}\right)-P D(e)$. (Again, $e \in H_{2}(X ; \mathbb{Z})$ generates the $\overline{\mathbb{C P}^{2}}$-summand.) 
Proof of Theorem 3.3. The application of Theorem 3.6 (together with the fact that $X^{\prime}$ is symplectic, hence admits nonvanishing Seiberg-Witten invariants) gives that

$$
S W_{X}\left(c_{1}(X, \omega)+2 P D(e)\right)= \pm 1
$$

hence by Theorem 3.4 we have that $G r_{X}(e) \neq 0$. This implies that the space $\mathfrak{H}(e, J)$ of $J$-holomorphic curves representing $e$ is nonempty. (In fact, Taubes proved that for a generic choice of $J$ the above space contains finitely many points, and (counted with appropriate multiplicities) the cardinality of $\mathfrak{H}(e, J)$ is equal to the SeiberWitten invariant $S W_{X}\left(c_{1}(X, \omega)+2 P D(e)\right)$.) The fact that $\mathfrak{H}(e, J)$ is nonempty implies that there is an element $\left\{\left(C_{k}, m_{k}\right)\right\}_{k=1}^{n} \in \mathfrak{H}(e, J)$ where $C_{k} \subset X$ is a $J$ holomorphic curve and $m_{k}$ is an appropriate multiplicity (which is one, unless $C_{k}$ is a torus with square 0$)$ with $\sum m_{k}\left[C_{k}\right]=e$. This equality implies that $\sum_{k=1}^{n}\left[C_{k}\right]^{2}=$ $e^{2}=-1$ and $c_{1}(X, \omega) \cdot \sum_{k=1}^{n}\left[C_{k}\right]=c_{1}(X, \omega) \cdot e=1$. (Note that tori with square 0 do not contribute to any of the above expressions; hence multiplicities can be disregarded.) Now $g\left(C_{k}\right)=1+\frac{1}{2}\left(\left[C_{k}\right]^{2}-c_{1}(X, \omega)\left[C_{k}\right]\right)$ and the above said implies that $\sum_{k=1}^{n} g\left(C_{k}\right)=n+\frac{1}{2}\left(\sum\left[C_{k}\right]^{2}-\sum c_{1}(X, \omega)\left[C_{k}\right]\right)=n-1$; hence there is at least one index $k$ for which $g\left(C_{k}\right)=0$, i.e., $C_{k}$ is a $J$-holomorphic sphere. This concludes the proof of Theorem 3.3

Proof of Theorem 1.4 As we mentioned before, if $f: X \rightarrow \Sigma$ is the blow-up of a torus bundle, then the classification of those bundles implies the result. (Also, we assume that $g(F)>0$; hence $b_{2}^{+}(X)>1$ holds.) Equip $X$ with a symplectic form $\omega$ (provided by the theorem of Gompf [G], GS]) and a compatible almost-complex structure $J$ for which $f$ is a pseudo-holomorphic map. Assume that the 4-manifold $X$ is not minimal, i.e., there is an embedded sphere $S \subset X$ with $[S]^{2}=-1$. Applying Theorem 3.3 for this sphere we find an embedding $i: \mathbb{C P}^{1} \hookrightarrow X$ such that the image is a $J$-holomorphic submanifold of $X$. Restrict $f$ to $i\left(\mathbb{C P}^{1}\right)$; the resulting map $\left.f\right|_{i\left(\mathbb{C P}^{1}\right)}: i\left(\mathbb{C P}^{1}\right) \rightarrow \Sigma$ is a pseudo-holomorphic map. Note that an almost-complex structure on a Riemann surface is always integrable. Consequently $\left.f\right|_{i\left(\mathbb{C P}^{1}\right)}$ is a holomorphic map between two complex curves; standard argument gives that in case the genus $g(\Sigma)$ is at least 1 , the above map $\left.f\right|_{i\left(\mathbb{C P}^{1}\right)}$ is constant. This, however, implies that the sphere $i\left(\mathbb{C P}^{1}\right)$ is in a fiber of the Lefschetz fibration $f: X \rightarrow \Sigma$. Since we assumed that $b_{2}^{+}(X)>1$, Corollary 3.5 and Lemma 3.1 imply that $\left[i\left(\mathbb{C P}^{1}\right)\right]=-1$, and since this sphere is in a fiber, the Lefschetz fibration $f: X \rightarrow \Sigma$ is not relatively minimal. Hence we found that if $X$ is nonminimal, then any Lefschetz fibration $f: X \rightarrow \Sigma$ with $g(\Sigma)>0$ is not relatively minimal, which proves Theorem 1.4.

Corollary 3.7. If $f: X \rightarrow \Sigma$ is a relatively minimal Lefschetz fibration and $g(F)$, $g(\Sigma)>0$, then $c_{1}^{2}(X) \geq 0$.

Proof. By Theorem 1.4 the manifold $X$ is minimal. Since $b_{2}^{+}(X)>1$, the theorem of Taubes [T2] (showing that a minimal symplectic 4-manifold $X$ with $b_{2}^{+}(X)>1$ has $c_{1}^{2}(X) \geq 0$; see also $[\underline{\mathrm{Ko}}$ ) applies, concluding the argument.

Proof of Theorem 1.5. Putting the above inequality and Corollary [2.6] together, we conclude that if $f: X \rightarrow \Sigma$ is a relatively minimal Lefschetz fibration with $g(F), g(\Sigma)>0$, then $0 \leq c_{1}^{2}(X) \leq 5 c_{2}(X)$, which proves the assertion of Theorem 1.5 . 
Remark 3.8. Note that most of the above arguments do not apply in the case when $g(\Sigma)=0$. There are plenty of examples of relatively minimal Lefschetz fibrations with nonminimal total spaces. (Take, for example, the rational elliptic surface: an elliptic fibration on the projective plane nine times blown up $\left(\approx \mathbb{C P}^{2} \# 9 \overline{\mathbb{C P}^{2}}\right)$.)

\section{ACKNOWLedgement}

The author would like to thank the Mathematics Research Center at the University of Warwick for their hospitality and the Magyary Zoltán Foundation and OTKA for their support.

\section{REFERENCES}

[BPV] W. Barth, C. Peters and A. Van de Ven, Compact complex surfaces, Ergebnisse der Mathematik, Spinger-Verlag Berlin, 1984. MR 86c:32026

[D1] S. Donaldson, An application of gauge theory to four dimensional topology, J. Diff. Geom. 18 (1983), 279-315. MR 85c:57015

[D2] S. Donaldson, Symplectic submanifolds and almost-complex geometry, J. Diff. Geom. 44 (1996), 666-705. MR 98h:53045

[F] M. Freedman, The topology of four-dimensional manifolds, J. Diff. Geom. 17 (1982), 357-453. MR 84b:57006

[FS1] R. Fintushel and R. Stern, Immersed spheres in 4-manifolds and the immersed Thomconjecture, Turkish J. Math. 19 (1995), 27-40. MR 96j:57036

[FS2] R. Fintushel and R. Stern, Rational blowdowns of smooth 4-manifolds, J. Diff. Geom. 46 (1997), 181-235. MR 98j:57047

[G] R. Gompf, Lecture at MSRI, $199 \%$.

[GS] R. Gompf and A. Stipsicz 4-Manifolds and Kirby Calculus, book in preparation.

[Ka] A. Kas, On the handlebody decomposition associated to a Lefschetz fibration Pacific J. Math. 89 (1980), 89-104. MR 82f:57012

[Ko] D. Kotschick, The Seiberg-Witten invariants of symplectic four-manifolds [after C. H. Taubes], Seminare Bourbaki 48 ème année (1995-96) no 812, 195-220. MR 98h:57057

$[\mathrm{KM}] \quad$ P. Kronheimer and T. Mrowka, The genus of embedded surfaces in the projective plane, Math. Res. Letters 1 (1994) 797-808. MR 96a:57073

[M] Y. Matsumoto, Diffeomorphism types of elliptic surfaces, Topology 25 (1986), 544-563. MR 88b:32061

[MSzT] J. Morgan, Z. Szabó and C. Taubes, A product formula for the Seiberg-Witten invariants and the generalized Thom conjecture, J. Diff. Geom. 44 (1996) 818-893. MR 97m:57052

[P] U. Persson, Chern invariants of surfaces of general type, Compositio Math. 43 (1981), 3-58. MR 83b:14012

[PPX] U. Persson, C. Peters and G. Xiao, Geography of spin surfaces, Topology 35 (1996), 845-862. MR 98h:14046

[S1] A. Stipsicz, A note on the geography of symplectic manifolds, Turkish J. Math. 20 (1996), 135-139. MR 97m:57035

[S2] A. Stipsicz, Simply connected symplectic 4-manifolds with positive signature, preprint.

[T1] C. Taubes, The Seiberg-Witten invariants and symplectic forms, Math. Res. Letters 1 (1994), 809-822. MR 95j:57039

[T2] C. Taubes, $S W \rightarrow G r:$ From the Seiberg-Witten equations to pseudo-holomorphic curves, Journal of the AMS 9 (1996), 845-918. MR 97a:57033

[T3] C. Taubes, Counting pseudo-holomorphic submanifolds in dimension 4, J. Diff. Geom. 44 (1996), 818-893. MR 97k:58029

[W] E. Witten, Monopoles and four-manifolds, Math. Res. Lett. 1 (1994), 769-796. MR 96d:57035

Department of Analysis, Elte TtK, 1088. Múzeum krt. 6-8., Budapest, Hungary

E-mail address: stipsicz@cs.elte.hu

Current address: Department of Mathematics, University of California, Irvine, California 92697-3875 